\title{
Erste Erfahrungen mit dem praktischen Einsatz von Qualitätsindikatoren
}

\section{J. Eckardt}

Qualitätsmanagement

Schlüssselvörter

Qualitätsindikatoren

Patientensicherheit

Routinedaten

DAHRQ

Helios-Qualitätskennzahlen

BOS-Qualitätsindikatoren

$\checkmark$ Reporting

Key words

quality indicators

routine data

reporting
Institut

Maria Hilf Kranken- und Pflegegesellschaft mbH, Dernbach

\section{Bibliografie}

Dol 10.1055/s-0028-1085590 Dtsch Med Wochenschr 2008. 133: S146 - @ Georg Thieme Verlag KG Stuttgart - New York . ISSN 0012-0472

\section{Korrespondenz}

Dr. med. Jörg Eckardt MHM

Maria Hilf Kranken- und Pflegegesellschaft mbH Katharina-Kasper-Straße 12 D-56428 Dernbach Tel. +49 2602/9301-641 eMail J.Eckardt@

Maria-Hilf-Dernbach.de
In Rheinland-Pfalz und Saarland haben sich 30 Einrichtungen zu einem Benchmarkingprojekt „Qualitätsindikatoren für katholische Krankenhäuser" zusammengeschlossen. Die teilnehmenden Einrichtungen gehören zu insgesamt 5 katholischen Trägern. Die durchschnittliche Einrichtungsgröße beträgt 283 Betten. Die 30 Einrichtungen verteilen sich auf die Cluster 50 bis 150 Betten (3), 151 bis 250 Betten (15), 251 bis 450 Betten (7) und 451 bis 700 Betten (5).

\section{Material und Methode}

Die Einrichtungen liefern Rohdaten im „§21 KHEntgG-Format“ und Daten der externen Qualitätssicherung (im Export-Format der Bundesgeschäftsstelle für Qualitätssicherung, $\mathrm{BQS}$ ). Für die Auswertung durch die Firma 3M Medica werden zurzeit folgende Indikatorensets herangezogen:

- Patient Safety Indicators (PSI) der Agency for Healthcare Research and Quality (AHRQ), 29 Indikatoren (Version 2.1, Revision 3);

- Helios-Qualitätskennzahlen (2. Generation), 78 Indikatoren;

- BQS-Qualitätsindikatoren ( $§ 137$ SGB V), 144 Indikatoren, 216 Kennzahlen (2006);

- optional: Qualitätssicherung mit Routinedaten (QSR-Projekt des AOK-WidO);

- optional: Projektspezifische Qualitätsindikatoren (u.U. Lebensqualitätsmessung).

Durch 3M erfolgen Risikoadjustierung und Berechnung der Erwartungswerte. Soweit Vergleichswerte des Statistischen Bundesamtes nicht zur Verfügung stehen, nutzt 3M einen eigenen Datenpool, in den je nach Jahrgang zwischen 3 und 3,5 Millionen Falldaten eingespielt sind. Die Einrichtungen erhalten quartalsweise Auswertungen zu den Qualitätsindikatoren in Form eines Datenwürfels und statische Reports. Halbjährlich werden die Leistungsdaten zu einem Managementreport aufbereitet.

Mit einer entsprechenden Version des Fileinspectors können die Einrichtungen ihre §21-Daten nicht nur überprüfen, sondern auch gemäß der Indikatorensets auswerten.

\section{Erfahrungen und Bewertung}

Die zur Verfügung gestellten Ergebnisse sind sehr umfangreich. Sie eignen sich unter dem Gesichtspunkt einer Ressourcen schonenden Vermittlung von entscheidungsrelevanten Informationen nicht zur direkten Weitergabe. Die verwendeten Indikatorensets von AHRQ Helios und BQS müssen verdichtet werden (auf Fachabtei- lungsebene oder je MDC), und es müssen in ihrer Wertigkeit gleichartige Indikatoren gegenübergestellt werden. Von ähnlichen Erfahrungen berichten andere Autoren [2].

Die Stichproben sind in Abhängigkeit des betrachteten Zeitraumes häufig zu klein, um statistische Aussagen machen zu können. Die Heliosgruppe kumuliert daher die Daten auf Einrichtungsebene für einen Betrachtungszeitraum von 2 Jahren und auf Konzernebene von einem Jahr [1]. Um die Daten als Steuerungsinstrument nutzen zu können, müssen die Daten regelmäßig ausgewertet werden, z.B. monatlich. Dazu empfiehlt der Autor die Verwendung von gleitenden Durchschnittswerten. Da die Fallzahlen je nach Disziplin und Indikator sehr unterschiedlich ausfallen, wäre die Möglichkeit, unterschiedlich lange Perioden $\mathrm{zu}$ parametrisieren (Halbjahr, Jahr, Doppeljahr), eine ideal Ergänzung. Die Indikatorensets decken nicht das Spektrum aller Fachdisziplinen ab. Neben einer Aggregation der Daten und Zusammenführung der Indikatorensets (s.o.) ist eine sinnvolle Ergänzung erforderlich.

Mit dem Neueinstieg in ein Bewertungssystem sind nicht sofort verwertbare Ergebnisse zu erzielen. Einflüsse der Dokumentation müssen kontinuierlich identifiziert und eliminiert werden. Ein im Kontext der Abrechnung übliches Dokumentationsverhalten kann im Rahmen der Fremdnutzung der Routinedaten die Ergebnisse verfälschen. Dabei spielt der unterschiedliche Gebrauch von Definitionen eine maßgebliche Rolle. Die Algorithmen, die bei der Auswahl einer Stichprobe Anwendung finden, sind zumeist nicht bekannt. Eine umfassende Transparenz würde ganz wesentlich zu einer höheren Akzeptanz des Systems beitragen.

In Einzelfällen konnten gravierende Auffälligkeiten wegen der geringen Fallzahl statistisch nicht bewertet werden. In diesen Fällen ist die Auseinandersetzung mit den Einzelfällen unumgänglich. Diese können Auslöser für die Durchführung von trägerinternen Peer Reviews sein.

Autorenerklärung: Der Autor erklärt, dass keine relevanten finanziellen Verbindungen in Bezug auf dieses Manuskript bestehen.

\section{Literatur}

1 Mansky T. Von der Qualitätssicherung zum Qualitätsmanagement. Vortragsfolien vom 18.06.2007, Folie 21. Trier: 2007

2 Stausberg J, Bartels C, Bobrowski C. Gewinnung von Managementinformationen aus der externen vergleichenden Qualitätssicherung. Med Klin 2007; 102 (7): 507-14 